\title{
Structural Stability of Planar Bimodal Linear Systems
}

\author{
Josep Ferrer, Marta Peña and Antoni Susín \\ Departament de Matemàtica Aplicada I, ETSEIB, Universitat Politècnica de Catalunya, Diagonal 647, 08028 \\ Barcelona, Spain
}

\begin{abstract}
We consider bimodal linear dynamical systems consisting of two linear dynamics acting on each side of a given hyperplane, assuming continuity along the separating hyperplane. Focusing in the planar case, we describe which of these systems are structurally stable.
\end{abstract}

Keywords: Piecewise linear system, structural stability.

PACS: $02.10 . \mathrm{Ud}, 02.30 . \mathrm{Hq}$

\section{INTRODUCTION}

Piecewise linear control systems have attracted the interest of the researchers in recent years by their wide range of applications, as well as by the possible theoretical approaches. See, for example, [1], [2], [3], [4], [5], [6], [7] and [8].

Bimodal linear systems consist of two subsystems acting on each side of a given hyperplane, assuming continuity along the separating hyperplane. We focus in the planar case. Indeed, it is very commonly found in applications (see the above references).

We adapt the conditions stated in [9] for piecewise-linear vector fields to the particular class of planar bimodal linear systems, describing which of these systems are structurally stable.

Throughout the paper, $\mathbb{R}$ will denote the set of real numbers, $M_{n \times m}(\mathbb{R})$ the set of matrices having $n$ rows and $m$ columns and entries in $\mathbb{R}$ (in the case where $n=m$, we will simply write $M_{n}(\mathbb{R})$ ) and $G l_{n}(\mathbb{R})$ the group of non-singular matrices in $M_{n}(\mathbb{R})$. Finally, we will denote by $e_{1}, \ldots, e_{n}$ the natural basis of the Euclidean space $\mathbb{R}^{n}$.

\section{PRELIMINARIES}

Let us consider a bimodal linear dynamical system given by

$$
\left\{\begin{array}{l}
\dot{x}(t)=A_{1} x(t)+B_{1}, \\
y(t)=C x(t),
\end{array} \quad \text { if } y(t) \leq 0, \quad\left\{\begin{array}{l}
\dot{x}(t)=A_{2} x(t)+B_{2}, \\
y(t)=C x(t),
\end{array} \quad \text { if } y(t) \geq 0\right.\right.
$$

where $A_{1}, A_{2} \in M_{n}(\mathbb{R}) ; B_{1}, B_{2} \in M_{n \times 1}(\mathbb{R}) ; C \in M_{1 \times n}(\mathbb{R})$. We assume that the dynamics is continuous along the separating hyperplane $H=\left\{x \in \mathbb{R}^{n}: C x=0\right\}$; that is to say, that both subsystems coincide for $y(t)=0$.

By means of a linear change in the state variable $x(t)$, we can consider $C=\left(\begin{array}{lll}1 & 0 \ldots 0\end{array}\right) \in M_{1 \times n}(\mathbb{R})$. Hence $H=\{x \in$ $\left.\mathbb{R}^{n}: x_{1}=0\right\}$ and continuity along $H$ is equivalent to:

$$
B_{2}=B_{1}, \quad A_{2} e_{i}=A_{1} e_{i}, \quad 2 \leq i \leq n .
$$

We will write from now on $B=B_{1}=B_{2}$.

Definition 1. In the above conditions, we say that the triple of matrices $\left(A_{1}, A_{2}, B\right)$ defines a bimodal linear dynamical system.

A natural tool is simplifying the matrices $A_{1}, A_{2}, B$ by means of changes in the variables $x(t)$ which preserve the qualitative behavior of the system. So, we consider linear changes in the state variables space preserving the hyperplanes $x_{1}(t)=k$, which will be called admissible basis changes. Thus, they are basis changes given by a matrix $S \in G l_{n}(\mathbb{R})$,

$$
S=\left(\begin{array}{cc}
1 & 0 \\
U & T
\end{array}\right), \quad T \in G l_{n-1}(\mathbb{R}), \quad U \in M_{n-1 \times 1}(\mathbb{R}) .
$$

Also, translations parallel to the hyperplane $H$ are allowed. 


\section{STRUCTURALLY STABLE PLANAR BIMODAL SYSTEMS}

By adapting the definition in [9] to our case we have:

Definition 2. A triple of matrices $\left(A_{1}, A_{2}, B\right)$ defining a bimodal linear dynamical system is said to be (regularly) structurally stable if it has a neighborhood $V\left(A_{1}, A_{2}, B\right)$ such that for every $\left(A_{1}^{\prime}, A_{2}^{\prime}, B^{\prime}\right) \in V\left(A_{1}, A_{2}, B\right)$ there is a homeomorphism of $\mathbb{R}^{2}$ preserving the hyperplane $H$ which maps the oriented orbits of $\left(A_{1}^{\prime}, A_{2}^{\prime}, B^{\prime}\right)$ into those of $\left(A_{1}, A_{2}, B\right)$ and it is differentiable when restricted to finite periodic orbits.

Our aim is to adapt the necessary and sufficient conditions in [9] to our triples:

$$
A_{1}=\left(\begin{array}{ll}
a_{1} & a_{3} \\
a_{2} & a_{4}
\end{array}\right), A_{2}=\left(\begin{array}{ll}
\gamma_{1} & a_{3} \\
\gamma_{2} & a_{4}
\end{array}\right), B=\left(\begin{array}{l}
b_{1} \\
b_{2}
\end{array}\right)
$$

Firstly, the singularities at infinity must be disjoint from the hyperplane $H$. Hence:

Lemma 1. Given a bimodal linear system defined by a triple $\left(A_{1}, A_{2}, B\right)$ as above, a necessary condition for it being structurally stable is $a_{3} \neq 0$.

Therefore, by means of a suitable admissible basis change (see [6]) and the translation $\left(0, b_{1}\right)$, we have:

Corollary 1. The triples representing a structurally stable bimodal linear system can be reduced to the form:

$$
\left(\begin{array}{ll}
a_{1} & 1 \\
a_{2} & 0
\end{array}\right),\left(\begin{array}{ll}
\gamma_{1} & 1 \\
\gamma_{2} & 0
\end{array}\right),\left(\begin{array}{c}
0 \\
b_{2}
\end{array}\right)
$$

Remark 1. The observable bimodal linear systems have a unique tangency point, which one has translated to the origin $(0,0)$. Notice also that foci (i.e., symmetrical nodes) are excluded.

Secondly, the conditions in [9] concerning finite singularities give:

Lemma 2. Given a bimodal linear system as in (*), necessary conditions for it being structurally stable are:

$$
\begin{aligned}
& \text { 1. } b_{2} \neq 0 \\
& \text { 2. } a_{2}>0 \text {, or } a_{2}<0 \text { and } a_{1} \neq 0 \\
& \text { 3. } \gamma_{2}>0 \text {, or } \gamma_{2}<0 \text { and } \gamma_{1} \neq 0
\end{aligned}
$$

Remark 2. The above lemma excludes the centers.

In particular, each subsystem has a unique equilibrium point (i.e., finite singularity). We say that is real if it is located in the semiplane corresponding to the considered subsystem. Otherwise, we say that the equilibrium point is virtual. The above lemma gives the following possible planar bimodal linear systems:

Corollary 2. The only bimodal linear systems verifying the necessary conditions in Lemma 1 and Lemma 2 are those in Table 1.

TABLE 1.

\begin{tabular}{ccccc}
\hline Subsystem $1 \backslash$ Subsystem 2 & Virtual saddle & Real node & Real spiral & Real improper node \\
\hline Real saddle & $1\left(b_{2}>0\right)$ & $2\left(b_{2}>0\right)$ & $3\left(b_{2}>0\right)$ & $4\left(b_{2}>0\right)$ \\
Virtual node & $5\left(b_{2}<0\right)$ & $6\left(b_{2}>0\right)$ & $7\left(b_{2}>0\right)$ & $8\left(b_{2}>0\right)$ \\
Virtual spiral & $9\left(b_{2}<0\right)$ & $10\left(b_{2}<0\right)$ & $11\left(b_{2}>0\right)$ & $12\left(b_{2}>0\right)$ \\
Virtual improper node & $13\left(b_{2}<0\right)$ & $14\left(b_{2}<0\right)$ & $15\left(b_{2}<0\right)$ & $16\left(b_{2}>0\right)$ \\
\hline
\end{tabular}

Finally, we must consider the conditions in [9] concerning finite orbits:

(a) the finite periodic orbits are hyperbolic and disjoint from the tangency points

(b) there are not finite orbits connecting two saddles

(c) there are not finite orbits connecting a saddle and a tangency point 
It is quite clear that finite periodic orbits can appear only in cases 3, 7, 11 and 15, whereas (b)-(c) only in case 3. Hence:

Proposition 1. The cases 1, 2, 4, 5, 6, 8, 9, 10, 12, 13, 14 and 16 are structurally stable.

Due to the limited space, we focus on case 3, leaving the remainder ones for future works.

Theorem 1. Let us consider the bimodal linear systems 3 in Corollary 2 with $\gamma_{1}>0$, that is to say:

$$
a_{2}>0, \gamma_{1}>0,0<\gamma_{1}^{2}<-4 \gamma_{2}, b_{2}>0
$$

1. The only saddel-loop orbit appears for $\gamma_{1}$ verifying

$$
\exp (\alpha t) \sin (\beta t-\varphi)+\frac{\beta}{M}=0, \quad \pi+\frac{\varphi}{\beta} \leq t \leq \frac{3 \pi}{2}+\frac{\varphi}{\beta}
$$

being

$$
t=\frac{1}{2 \alpha} \ln \left(\frac{\alpha^{2}+\beta^{2}+\lambda_{1}^{2}-2 \lambda_{1} \alpha}{\lambda_{1}^{2}\left(1-2 \frac{\alpha}{\lambda_{2}}+\frac{\alpha^{2}+\beta^{2}}{\lambda_{2}^{2}}\right)}\right)
$$

where

$\lambda_{1}>0, \lambda_{2}<0$ are the eigenvalues of the saddle,

$\alpha \pm i \beta, \alpha, \beta>0$ are the eigenvalues of the spiral,

$M \cos (\varphi)=\alpha-\frac{\alpha^{2}+\beta^{2}}{\lambda_{2}}, \quad M \sin (\varphi)=\beta$

2. For minor values of $\gamma_{1}$, a finite periodic orbit appears, which is hyperbolic and disjoint from the tangency points.

3. For these values of $\gamma_{1}$, no saddle-tangency orbits appear.

Example 1. We show the case $a_{1}=-1, a_{2}=1, \gamma_{1}=0.1, \gamma_{2}=-5, b_{2}=1$ in Figure 1. We plot the phase portrait corresponding to the Poincaré map on the section $x=0$ for different initial points: for each of them the orbits are integrated until the next oriented cut. The continuous lines correspond to inward spiraling orbits and the discontinuous lines to outward spiraling ones. An hyperbolic finite periodic orbit exists between them.

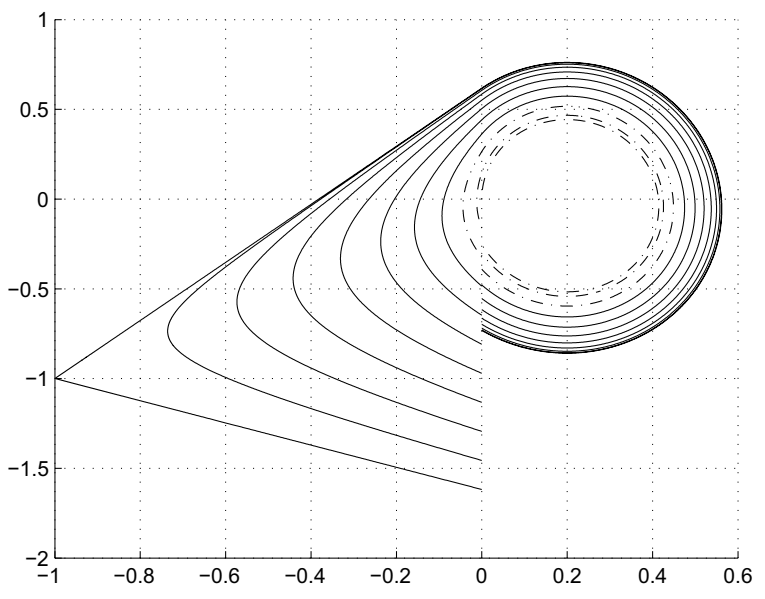

FIGURE 1.

The proof is based on the following lemmas:

Lemma 3. Let us assume that a finite periodic orbit exists. Then

$$
\frac{A^{+}}{A^{-}}=-\frac{a_{1}}{\gamma_{1}}
$$

where $A^{+}, A^{-}$are the enclosed areas in the right and the left side respectively. 
Lemma 4. Let us consider the saddle-spiral orbit passing through $\left(0,-\frac{b_{2}}{\lambda_{2}}\right)$. Then its first intersection with the separating hyperplane (if exists) is determined by

$$
\exp (\alpha t) \sin (\beta t-\varphi)+\frac{\beta}{M}=0, \quad \pi+\frac{\varphi}{\beta} \leq t \leq \frac{3 \pi}{2}+\frac{\varphi}{\beta}
$$

Corollary 3. The systems in Theorem 1 with $\gamma_{1}$ as in 2./3. are structurally stable.

Remark 3. A homoclinic (i.e., saddle-loop) orbit bifurcation appears when 1. in Theorem 1 occurs.

\section{REFERENCES}

1. Artes, J., Llibre, J., Medrado, J.C. \& Teixeira, M.A., Piecewise linear differentail systems withtwo real saddles, Mathematics and Computers in Simulation, 2013.

2. CAMLibel, K., HeEmels, M. \& SCHUMACHER, H., Stability and controllability of planar bimodal linear complementarity systems, Proceedings of the 42nd IEEE Conference on Decision and Control, 1651-1656, 2003.

3. Camlibel, K., Heemels, M. \& SChumacher, H., On the controllability of bimodal piecewise linear systems, LNCS 2993, 250-264, 2004.

4. Camlibel, K., Heemels, M. \& Schumacher, H., A full characterization of stabilizability of bimodal piecewise linear systems with scalar inputs, Automatica, 44, 1261-1267, 2008.

5. Di Bernardo, M., Pagano, D. J. \& POnCE, E., Nonhyperbolic boundary equilibrium bifurcations in planar Filippov systems: a case study approach, J. Bifur. Chaos Appl. Sci. Engin., 18, 1377-1392, 2008.

6. Ferrer, J., Magret, M. \& PeÑA, M., Bimodal piecewise linear systems. Reduced forms, International Journal of Bifurcation and Chaos, 20, 2795-2808, 2010.

7. Ferrer, J., PACHA, J. R. \& PeÑA, M., Controllability of continuous bimodal linear systems, Mathematical Problems in Engineering (special issue Mathematical Modeling, Analysis, and Control of Hybrid Dynamical Systems), Volume 2013 (2013), Article ID 342548, 14 pages.

8. Llibre, J., Ordonez, M. \& Ponce, E., On the existence and uniqueness of limit cycles in planar continuous piecewise linear systems without symmetry, Nonlinear Analysis: Real World Applications, 2013.

9. Sotomayor, J. \& Garcia, R., Structural stability of piecewise-linear vector fields, Journal of Differential Equations, 192, 553-565, 2003. 